\title{
Assessing the usefulness and acceptability of a low health literacy online decision aid about reproductive choices for younger women with breast cancer: the aLLIAnCE pilot study protocol
}

Michelle Peate ${ }^{1 *}$ (D), Sian Karen Smith², Victoria Pye ${ }^{3}$, Alice Hucker ${ }^{1}$, Catharyn Stern ${ }^{4}$, Lesley Stafford ${ }^{5}$, Catherine Oakman ${ }^{6}$, Laura Chin-Lenn ${ }^{6}$, Kerry Shanahan ${ }^{7}$, Nipuni Ratnayake Gamage ${ }^{1}$ and Martha Hickey ${ }^{8}$

\begin{abstract}
Background: Young women diagnosed with breast cancer may be confronted by many difficult decisions, especially around fertility preservation prior to commencing cancer treatment. The information to be conveyed is complex, and it may be difficult to weigh up the risks and benefits of the different fertility preservation options available. This complexity is compounded by the widespread low levels of literacy and health literacy in Australia, which may result in greater difficulties in understanding available health information and in decision-making.

Methods/design: A working group of experts have developed a fertility-related online decision aid for a low health literacy population, guided by health literacy principles. The decision aid will be pilot tested with 30 women diagnosed with early breast cancer between 5 years and 6 months previously. To be eligible, at the time of diagnosis, women must be between 18 and 40 years (inclusive), pre-menopausal, have no history of metastatic disease, have not completed their families, be able to give informed consent and have low health literacy. Participants will be asked to reflect back to the time in which they were diagnosed. Participants will complete a questionnaire before and after reviewing the decision aid to determine the feasibility, use and acceptability of the decision aid. The decision aid will be modified accordingly. Participants may also choose to review a previously developed (high literacy) decision aid and provide feedback in comparison to the low health literacy decision aid.

Discussion: This project represents the first study to develop an online fertility decision aid developed from low health literacy models in the context of breast cancer. It is anticipated that the low health literacy decision aid will be useful and acceptable to young women with low health literacy who have been diagnosed with breast cancer and that it will be preferred over the high literacy decision aid.
\end{abstract}

Trial registration: ACTRN12615001364561p

Keywords: Breast cancer, Fertility, Fertility preservation, Decision-making, Young women, Decision aid, Low health literacy, Psychosocial issues, Unmet needs

* Correspondence: mpeate@unimelb.edu.au

${ }^{1}$ Psychosocial Health and Wellbeing Research (emPoWeR) Unit, Department

of Obstetrics \& Gynaecology, University of Melbourne, Level 7, Royal Women'

Hospital, 20 Flemington Road, Parkville, VIC 3052, Australia

Full list of author information is available at the end of the article

(c) The Author(s). 2017 Open Access This article is distributed under the terms of the Creative Commons Attribution 4.0 International License (http://creativecommons.org/licenses/by/4.0/), which permits unrestricted use, distribution, and reproduction in any medium, provided you give appropriate credit to the original author(s) and the source, provide a link to the Creative Commons license, and indicate if changes were made. The Creative Commons Public Domain Dedication waiver (http://creativecommons.org/publicdomain/zero/1.0/) applies to the data made available in this article, unless otherwise stated. 


\section{Background}

Breast cancer is the most frequently diagnosed cancer in reproductive aged women $[1,2]$. In Australia, approximately 1000 women annually are under 40 years of age at the time of diagnosis [1]. Prognosis is often excellent, but adjuvant chemotherapy commonly results in permanent ovarian failure, depending on age at the time of treatment and dose and type of chemotherapy used [3]. Furthermore, delaying pregnancy until completion of endocrine therapy will be associated with an age-related reduction in fertility and may result in women being past their reproductive age by completion of treatment. Additionally, as women are starting their families at older ages than in previous decades [4], breast cancer in women who are childless or have not completed their families at diagnosis is becoming more common. For many, the impact of cancer treatment on future fertility is a significant issue [5].

Fortunately, for many young women, there are fertility preservation options available. It is essential that women have access to high-quality information in order to make decisions about these interventions. Previous research has found that many young women are not fully informed [5-8]. Patients who are better informed experience greater emotional, social and physical well-being [9]; better clinical outcomes; better quality of life [9]; and improved satisfaction with care [10]. Thus, good management must include consideration of fertility information needs [11].

To address these needs, a fertility decision aid that improved knowledge and satisfaction while reducing uncertainty and regret has been previously developed [12-14]. However, there is already growing evidence of poor outcomes for people with low health literacy with the use of decision aids that do not target this group [15]. In a systematic review of patient decision aid trials, lower health literacy was associated with lower patient health knowledge, higher decisional uncertainty and regret and lower desire for involvement in the decision-making process and less question-asking and less patient-centred communication [15]. Considering this evidence of poorer outcomes and that $52 \%$ of Australian women aged 15 to 44 years do not have the minimum literacy skills required to 'meet the complex demands of everyday life' [16], it is highly likely this population will not benefit from the existing decision aid. Specifically, the decision aid does not align with the updated International Patient Decision Aids Standards (IPDAS) recommendations for low health literacy as the language of the existing textdense decision aid was not written at a level that would be understood by the majority of a low health literacy group, nor was it developed using good health literacy principles [15]. As such, there is an argument that provision of information that is not targeted for low health literacy is inappropriate, especially in the context of making informed decisions $[17,18]$.

Health literacy is defined as the knowledge and skills required to understand and use information relating to health issues [16]. Those with low health literacy may lack the cognitive and social skills that determine the capacity to gain access to, understand and use information to support good health [19]. They are often disempowered because they receive less information, ask fewer questions and are less satisfied with health care provider communication [20]. Furthermore, low health literacy is associated with a wide range of adverse health outcomes, including reduced overall health status and poorer selfcare management $[21,22]$. Thus, low health literacy groups, such as culturally and linguistically diverse and indigenous groups, are socially disadvantaged. Additionally, even people who are usually considered to have high literacy may have low health literacy when faced with a difficult diagnosis [23]. It is hoped that targeted information will help those in this low health literacy group to cope with the information overload that often occurs [24]. Few decision aids have been developed for low health literacy populations, and this is a recognised gap in consumer resources $[17,25]$.

Recently, a Dutch fertility decision aid was developed for women with breast cancer with lower education [26] and found that the improvement in knowledge of less educated women was less than the improvement seen in the better educated women, after receiving the decision aid [27]. Although the Dutch decision aid showed differences in outcomes according to education level, it did not specifically target or investigate low health literacy. Considering that women with high education can also have low health literacy, the Dutch tool does not address the issues beyond level of language as it was not designed according to the IPDAS low health literacy guidelines. It is not merely the level of language that is important but also the reading, listening, analytical and decision-making skills [28, 29] which must be addressed when developing materials to improve patient understanding. Online interventions are a useful medium for this purpose as it allows for alternative formats for information delivery (other than just text) and can be used for interactive learning, such as teach-back methodology, which have been shown to improve satisfaction and have beneficial effects on health behaviours [15]. Thus, there is an urgent need to develop a low health literacy fertility decision aid developed using low health literacy strategies that can be accessed and understood by all patients.

As the discussion of fertility preservation is recommended at the highly stressful time of cancer diagnosis $[30,31]$, it is important to evaluate the impact of decision aids [32] to ensure that they do not make an already 
difficult situation worse. Thus, the aim of this pilot study is to develop and assess the acceptability of a low literacy fertility-related decision aid for young women with early breast cancer and low health literacy. The goal is to ensure that the decision aid would be useful at the time of their diagnosis, would improve their knowledge and would not have a negative emotional impact.

\section{Methods/design}

A two-stage process will be used to ensure that the decision aid is acceptable to patients and is an effective decision tool. This paper reports on the protocol for the development and pilot testing of the decision aid.

The decision aid has been designed in accordance with the latest IPDAS [14] to assist patients with low health literacy reach an informed decision about fertility preservation. Although the aim in this study is to establish acceptability in a low health literacy population, we anticipate this will also be useful for all women (and this will be evaluated in the next phase of the study). It includes information concerning the pros and cons of fertility preservation in women with early breast cancer and has evidence-based representation of chances of success. It addresses the complexity, uncertain benefits and potentially large costs (e.g. emotional, financial and physical) in this setting. A summary of treatment procedures is presented with a set of value-clarification exercises to help weigh up the pros and cons of fertility preservation in light of patient values and life situation.

The decision aid is web-based to allow for the use of various communication forms. It is specifically designed for a low health literacy audience and includes illustrations, audio and video components to communicate topics and enhance understanding [33]. Text has been simplified and lay language used. The content is based on the previously developed fertility-related decision aid $[12,34,35]$, a literature review and consultation with experts, including health literacy experts, oncologists, breast surgeons, breast care nurses, reproductive health specialists, psychologists, behavioural scientists and consumers. It is guided by basic education and linguistic theory, health literacy and risk communication research, and the conceptual models of low health literacy, to maximise comprehension [29, 36-38].

\section{Aim}

The aim of this study is to assess the usefulness and acceptability of a low health literacy decision aid for fertility preservation amongst young women with early breast cancer. Additionally, a secondary outcome for women is to assess whether the decision aid improves knowledge.

\section{Sample size}

Recommended sample sizes for pilot studies are 10-30 participants [39-42] or $10 \%$ of the sample projected for the larger parent study [43, 44]. As our final goal in stage two (described below) is to recruit 270 women for a final sample of 178, the pilot will comprise 30 women.

\section{Eligibility criteria}

Women with a previous diagnosis of early breast cancer will be recruited. To be eligible to participate, women must, at the time of diagnosis:

(a) Be aged between 18 and 40 years (inclusive)

(b) Have had a histologically confirmed diagnosis of early-stage breast cancer

(c) Be pre-menopausal (regular menstrual periods and no vasomotor symptoms)

(d)Have had no history of metastatic disease

(e) Have not completed their families

\section{And currently:}

(f) Diagnosed between 6 months and 5 years previously; the lower limit of 6 months was chosen to avoid increased burden on women who are currently on active treatment, and the upper limit of 5 years was selected primarily as most young women with breast cancer are advised to avoid pregnancy for at least 2 years and for some up to 5 years (upon completion of endocrine therapy) and these women may still be thinking about having children. This time window also ensures that we would have the participant numbers from our two sites to provide useful pilot data.

(g) Be able to give informed consent.

(h)Be identified as having low health literacy using the Newest Vital Sign (NVS) health literacy instrument as a screening tool (a 5-min food label quiz structured around reading and understanding information) [45].

Women will also need adequate English skills to complete the questionnaires and communicate with the researchers.

\section{Procedure}

Pilot testing will be conducted through the oncology clinic at a tertiary-level hospital in a major metropolitan centre following the process outlined in Fig. 1. The decision aid will be modified based on the findings from the pilot testing. 


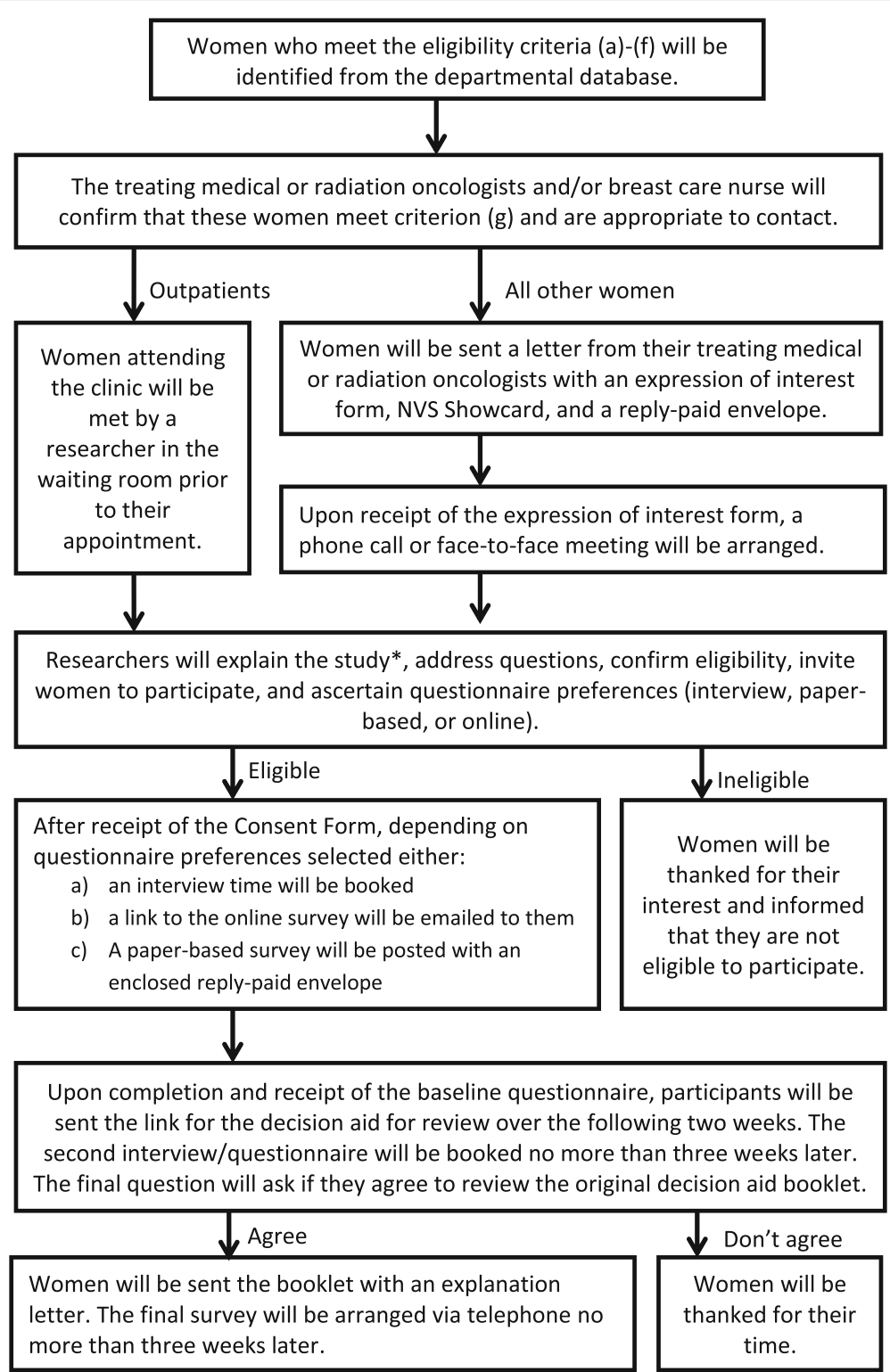

Fig. 1 Stage 1 pilot-testing procedure. *The voluntary nature of participation will be explained, including that declining participation or later withdrawal will not impact on medical care

\section{Questionnaires/measures}

We will be adapting a questionnaire previously used in related studies to evaluate patient decision support education tools to be suitable for a low health literacy population [34]. Two questionnaires will be administered pre- and post-review of the decision aid. A third, brief, questionnaire will be administered to participants who opt in at the end of the second questionnaire to compare this online decision aid to the original paperbased (high literacy) decision aid [12]. Participants will be given the option to complete the questionnaire online, on a paper questionnaire or via interview. The items in these questionnaires are shown in Table 1.

\section{Analysis}

Basic descriptive statistics, including means, medians, percentages, ranges and standard deviations, and 95\% confidence intervals, will be calculated to describe the sample in terms of socio-demographic characteristics. The comparison survey will be analysed descriptively.

\section{Discussion}

Fertility concerns continue to be an important issue for young women diagnosed with breast cancer. These decisions are often made under pressure of time and may be complex, especially as it is a rapidly changing field. This is compounded by the widespread low levels of literacy 
Table 1 Pilot-testing questionnaire content (stage 1)

\begin{tabular}{|c|c|c|c|}
\hline & Pre & Post & Comparison survey \\
\hline $\begin{array}{l}\text { Demographic data, including current age, age at diagnosis, } \\
\text { relationship status, postcode of residence, country of birth, } \\
\text { time lived in Australia, Aboriginal or Torres Strait Islander origin, } \\
\text { first language spoken, level of English, highest education level, } \\
\text { medical and allied health training, employment status, profession, } \\
\text { parity and menopausal status. }\end{array}$ & $\checkmark$ & & \\
\hline $\begin{array}{l}\text { Importance of fertility at time of diagnosis (very, somewhat, not at all) } \\
\text { and plans for future children (yes/no, when). }\end{array}$ & $\checkmark$ & & \\
\hline $\begin{array}{l}\text { Data on cancer treatment and fertility specialist referrals for previous } \\
\text { cancer treatment, as well as fertility treatments accessed. }\end{array}$ & $\checkmark$ & & \\
\hline $\begin{array}{l}\text { Knowledge of breast cancer treatment and fertility preservation } \\
\text { assessed using } 10 \text { items from a knowledge scale previously used [12], } \\
\text { adapted for people with low health literacy. Correct responses will be } \\
\text { summed for a knowledge score. }\end{array}$ & $\checkmark$ & $\checkmark$ & \\
\hline $\begin{array}{l}\text { Acceptability: how the decision aid was accessed (time spent working } \\
\text { through materials, thoroughness) and perceptions about the content } \\
\text { (amount of information, length, presentation, appeal, ease of reading, } \\
\text { order of topics, pace, balance, confusion, direction, clarity). }\end{array}$ & & $\checkmark$ & \\
\hline $\begin{array}{l}\text { Acceptability: how the decision aid was accessed (thoroughness) and } \\
\text { perceptions about the content (presentation, appeal, ease of reading, } \\
\text { clarity). }\end{array}$ & & & $\checkmark$ \\
\hline $\begin{array}{l}\text { Perceived improvement in understanding: six items specifically designed } \\
\text { to assess perceived improvement in understanding of the impact of } \\
\text { cancer treatment on fertility and of fertility treatment on the cancer } \\
\text { prognosis and the pros and cons associated with each available fertility } \\
\text { option. Response options ranging from 'not at all' to 'a lot'. }\end{array}$ & & $\checkmark$ & \\
\hline $\begin{array}{l}\text { Satisfaction with the decision aid: evaluating the amount of information, } \\
\text { length, balance, direction and presentation of the decision aid using } \\
\text { structured response categories. Also, specific questions regarding the } \\
\text { ease of understanding of particular sections of the decision aid will } \\
\text { be included. }\end{array}$ & & $\checkmark$ & \\
\hline $\begin{array}{l}\text { Emotional impact of the decision aid: if the decision aid (or specific } \\
\text { sections of the decision aid) is reassuring, causes worry, concern or } \\
\text { distress, and whether the decision aid would have helped them cope } \\
\text { better with their situation. Response options ranging from "not at all" } \\
\text { to "very much so". }\end{array}$ & & $\checkmark$ & \\
\hline $\begin{array}{l}\text { Relevance of the decision aid: perceived relevance of the decision aid } \\
\text { will be determined using two Likert-style questions asking participants } \\
\text { to indicate how relevant they felt the information would have been at } \\
\text { the time of diagnosis and how helpful the decision aid would have been } \\
\text { in reaching their decision. One item will assess whether participants } \\
\text { would recommend the decision aid to others in the same situation. }\end{array}$ & & $\checkmark$ & \\
\hline $\begin{array}{l}\text { Open-ended questions: space will be provided for participants to } \\
\text { comment on their satisfaction with the decision aid, the relevance of } \\
\text { the decision aid to their situation and the emotional impact of the } \\
\text { decision aid. Women will also be asked to identify areas which require } \\
\text { more or less detail, their preferred order of topics and for their general } \\
\text { suggestions for improvement. }\end{array}$ & & $\checkmark$ & \\
\hline $\begin{array}{l}\text { Comparison of decision aids: selection of which decision aid was more } \\
\text { helpful and why; selection of which decision aid was easier to read } \\
\text { and understand and why; overall preferred decision aid and why. }\end{array}$ & & & $\checkmark$ \\
\hline
\end{tabular}

in Australia, which may result in greater difficulties in understanding available health information and in decision-making.

Women require decisional support in this area [8]. This has been addressed through the development of interventions such as decision aids [12, 27, 34]; however, these interventions are unlikely to cater for the large proportion of women with low health literacy [16]. The lack of decision support widens the disadvantage gap, with these women unlikely to have their information needs met. Thus, there is a need for tools that are specifically targeted to this population [17].

Additionally, the decision aid may be of benefit to women who are not identified as having low health 
literacy. Women faced with a breast cancer diagnosis are often feeling overwhelmed and need to make a number of important treatment decisions in a relatively short amount of time, and thus, a decision aid that supports the processing of complex information in a simple manner may be appropriate for all women in this group.

This project will represent the first study to develop a fertility decision aid that supports women with low health literacy to be actively involved in their health care decisions about fertility preservation in the context of breast cancer. The evidence-based tool was developed by experts in this field according to international guidelines for decision aids [14] and using low health literacy models. This pilot study will evaluate the acceptability and perceived usefulness of this decision aid amongst young women with a previous history of breast cancer to provide support for the prospective use of this tool. It is anticipated that not only will this decision aid be acceptable and useful to women but it also will improve knowledge without having negative emotional impact, and they would recommend the decision aid to others in a similar situation. It is also anticipated that participants will report the low health literacy decision aid as more useful and acceptable than the original tool. Future steps will include the prospective evaluation of the low health literacy decision aid in a cluster randomised controlled trial, followed by implementation of the decision aid into clinical practice.

\section{Abbreviations}

aLLIAnCE: Low Literacy Decision Aid about Reproductive Choices for Younger Women with Breast Cancer; IPDAS: International Patient Decision Aid Standards; NVS: Newest Vital Sign health literacy instrument

\section{Acknowledgements}

This research and Michelle Peate are supported by a grant from the National Breast Cancer Foundation (ECF-15-005). Martha Hickey is supported by a Practitioner Fellowship from the National Health and Medical Research Council (APP1058945). Sian Smith is supported by an Early Career Fellowship from the National Health and Medical Research Council (no. 1034912). This is an aLLIAnCE (Low Literacy Decision Aid about Reproductive Choices for Younger Women with Breast Cancer) Collaborative Group Study.

\section{Funding}

This research and Michelle Peate are supported by a grant from the National Breast Cancer Foundation (ECF-15-005).

\section{Availability of data and materials}

Not applicable.

\section{Authors' contributions}

MP conceived this study; participated in the design of the study, development of all study documents (including the decision aid), ethical approval process and study coordination; and drafted this manuscript. SS participated in the design of the study and in the development of all study documents (including the decision aid). VP carried out the sample size and power analysis and developed the statistical analysis plan. AH participated in the development of all study documents (including the decision aid), study databases, ethical approval process and study coordination. CS participated in the review of study documents and the development of the decision aid. LS and KS participated in the review of study documents and the development of the decision aid. CO participated in the review of study documents, ethical approval process, and the development of the decision aid. LCL participated in the review of study documents and the development of the decision aid. NRG participated in the review of study documents and helped to develop the study databases and the development of the decision aid. MH participated in the design of the study, development of the protocol, the development of the decision aid and the review of study documents. All authors read and approved the final manuscript.

\section{Competing interests}

The authors declare that they have no competing interests.

\section{Consent for publication \\ Not applicable.}

\section{Ethics approval and consent to participate}

This study has been approved by the tertiary-level Melbourne Health Human Research Ethics Committee (HREC) — AU RED HREC Reference Number: $\mathrm{HREC} / 15 / \mathrm{MH} / 263$.

\section{Universal Trial Number}

Universal Trial Number (UTN): U1111-1170-1252.

\section{Publisher's Note}

Springer Nature remains neutral with regard to jurisdictional claims in published maps and institutional affiliations.

\section{Author details}

'Psychosocial Health and Wellbeing Research (emPoWeR) Unit, Department of Obstetrics \& Gynaecology, University of Melbourne, Level 7, Royal Women' Hospital, 20 Flemington Road, Parkville, VIC 3052, Australia. ${ }^{2}$ Psychosocial Research Group, Prince of Wales Clinical School, Faculty of Medicine, UNSW, Lowy Research Centre C25, Level 4, Sydney, NSW 2052, Australia. ${ }^{3}$ Australian Institute of Health Innovation, Macquarie University, Sydney, NSW 2109, Australia. ${ }^{4}$ Endocrine and Metabolic Service and Reproductive Services, Royal Women's Hospital and Melbourne IVF, 20 Flemington Road, Parkville, VIC 3052, Australia. ${ }^{5}$ Centre for Women's Mental Health, The Royal Women's Hospital, 20 Flemington Road, Parkville, VIC 3052, Australia. 'Breast Service, Royal Melbourne and Royal Women's Hospitals, Parkville, VIC 3052, Australia. ${ }^{7}$ Breast Service, Royal Melbourne Hospital, Parkville, VIC 3052, Australia. ${ }^{8}$ Department of Obstetrics \& Gynaecology, University of Melbourne, Level 7, Royal Women's Hospital, 20 Flemington Road, Parkville, VIC 3052, Australia.

Received: 11 August 2016 Accepted: 11 May 2017

Published online: 07 June 2017

\section{References}

1. Australian Institute of Health and Welfare (Aihw) and Australasian Association of Cancer Registries (Aacr), Cancer in Australia 2001, in AlHW cat. no. CAN 23. 2004, AlHW (Cancer Series no. 28): Canberra.

2. Sonmezer M, Oktay K. Fertility preservation in female patients. Hum Reprod Update. 2004;10:251-66.

3. Minton S, Munster N. Chemotherapy-induced amenorrhea and fertility in women undergoing adjuvant treatment for breast cancer. Cancer Control. 2002:9:466-72.

4. Hammarberg K, Clarke VE. Reasons for delaying childbearing-a survey of women aged over 35 years seeking assisted reproductive technology. Aust Fam Physician. 2005;34:187-8. 206.

5. Peate M, Meiser B, Friedlander M, Zorbas H, Rovelli S, Sansom-Daly U, et al. It's now or never: fertility-related knowledge, decision-making preferences, and treatment intentions in young women with breast cancer-an Australian fertility decision aid collaborative group study. J Clin Oncol. 2011; 29:1670-7.

6. Duffy C, Allen M, Clark M. Discussions regarding reproductive health for young women with breast cancer undergoing chemotherapy. J Clin Oncol. 2005;20:766-73.

7. National Breast Cancer Foundation. Breast cancer research in Australia: meeting the challenges. Sydney: National Breast Cancer Foundation; 2004

8. Peate M, Meiser B, Hickey M, Friedlander M. The fertility-related concerns, needs and preferences of younger women with breast cancer: a systematic review. Breast Cancer Res Treat. 2009;116:215-23. 
9. Fallowfield $\sqcup$, Hall A, Maguire P, Baum M, A'hern RP. Psychological effects of being offered choice of surgery for breast cancer. Br Med J. 1994;309:448.

10. Weiss SM, Wengert Jr PA, Martinez EM, Sewall W, Kopp E. Patient satisfaction with decision-making for breast cancer therapy. Ann Surg Oncol. 1996:3:285-9.

11. National Breast Cancer Centre. Clinical practice guidelines for the management and support of younger women with breast cancer. Sydney: National Breast Cancer Centre; 2003.

12. Peate M, Meiser B, Cheah B, Saunders C, Butow P, Thewes B, et al. Making hard choices easier: a prospective, multicentre study to assess the efficacy of a fertility-related decision aid in young women with early-stage breast cancer. Br J Cancer. 2012;106:1053-61.

13. Butow $P$, Brindle E, Mcconnell D, Boakes R, Tattersall M. Information booklets about cancer: factors influencing patient satisfaction and utilisation. Pat Educ Counsel. 1998:33:129-41.

14. Elwyn G, O'connor A, Stacey D, Volk R, Edwards A, Coulter A, et al. Developing a quality criteria framework for patient decision aids: online international Delphi consensus process. BMJ. 2006;333:417.

15. Mccaffery KJ, Sheridan S, Nutbeam D, Clayman ML, Kelly-Blake K, Rovner D, et al., Addressing health literacy. In: Volk R \& Llewellyn-Thomas H, Eds. Update of the International Patient Decision Aids Standards (IPDAS) Collaboration's Background Document. 2012

16. Australian Bureau of Statistics. 4233.0 - Health Literacy, Australia, 20062008 24 July 2008 [cited 2014]. Available from: http://www.abs.gov.au/AUSSTATS/ abs@.nsf/Latestproducts/4233.0Main\%20Features22006.

17. Mccaffery KJ, Holmes-Rovner M, Smith SK, Rovner D, Nutbeam D, Clayman $\mathrm{ML}$, et al. Addressing health literacy in patient decision aids. BMC Med Inform Decis Mak. 2013;13:S10.

18. Mccray AT. Promoting health literacy. J Am Med Inform Assoc. 2005;12:152-63.

19. World Health Organization. Track 2: health literacy and health behaviour. 7th Global Conference on Health Promotion: track themes [cited 2014]. Available from: http://www.who.int/healthpromotion/conferences/7gchp/ track2/en/.

20. Ishikawa H, Yano E, Fujimori S, Kinoshita M, Yamanouchi T, Yoshikawa M, et al. Patient health literacy and patient-physician information exchange during a visit. Fam Pract. 2009;26:517-23.

21. Nutbeam D. Health literacy as a public health goal: a challenge for contemporary health education and communication strategies into the 21st century. Health Promot Int. 2000;15:259-67.

22. Berkman N, Sheridan S, Donahue K, Halpern D, Crotty K. Low health literacy and health outcomes: an updated systematic review. Ann Intern Med. 2011; 155:97-107.

23. Australian Commission on Safety and Quality in Health Care (Acsqhc). Health literacy: taking action to improve safety and quality. Sydney: ACSOHC; 2014.

24. Kyunghye K, Lustria MLA, Burke D, Kwon N. Predictors of cancer information overload: findings from a national survey. Inf Res. 2007;12:1.

25. Durand M-A, Carpenter L, Dolan H, Bravo P, Mann M, Bunn F, et al. Do interventions designed to support shared decision-making reduce health inequalities? A systematic review and meta-analysis. PLOS ONE. 2014;9:e94670.

26. Borstkanker en kinderwens ("Breast cancer and have children") website. Available from: http://www.borstkankerenkinderwens.nl.

27. Garvelink M, Ter Kuile M, Fischer M, Louwé L, Hilders C, Kroep J, et al. Development of a decision aid about fertility preservation for women with breast cancer in The Netherlands. J Psychosom Obstet Gynaecol. 2013;34:170-8.

28. National Network of Libraries. Health literacy. 2007. https://nnlm.gov/ professional-development/topics/health-literacy.

29. Sørensen K, Van Den Broucke S, Fullam J, Doyle G, Pelikan J, Slonska Z, et al. Health literacy and public health: a systematic review and integration of definitions and models. BMC Public Health. 2012;12:80

30. Lee SJ, Schover LR, Partridge AH, Patrizio P, Wallace WH, Hagerty $K$, et al. American Society of Clinical Oncology recommendations on fertility preservation in cancer patients. J Clin Oncol. 2006;24:2917-31.

31. Loren AW, Mangu PB, Beck LN, Brennan L, Magdalinski AJ, Partridge AH, et al. Fertility preservation for patients with cancer: American Society of Clinical Oncology Clinical Practice Guideline Update. J Clin Oncol. 2013;31:2500-10.

32. Stacey D, Légaré $F$, Col NF, Bennett $C L$, Barry MJ, Eden KB, et al. Decision aids for people facing health treatment or screening decisions. Cochrane Database Syst Rev. 2014
33. Houts $P$, Doak C, Doak L, Loscalzo M. The role of pictures in improving health communication: a review of research on attention, comprehension, recall, and adherence. Patient Educ Couns. 2006;64:393-4.

34. Peate M, Meiser B, Friedlander M, Saunders C, Martinello R, Wakefield C, et al. Development and pilot testing of a fertility decision aid for young women diagnosed with early breast cancer. Breast J. 2011;17:112-4.

35. Peate M, Butow P, Fallon-Ferguson J, Friedlander M, Hart R, Hickey M, et al. Fertility-related choices - a decision aid for younger women diagnosed with breast cancer. Randwick: Prince of Wales Clinical School; 2008.

36. Clerehan R, Buchbinder R, Moodie J. A linguistic framework for assessing the quality of written patient information: its use in assessing methotrexate information for rheumatoid arthritis. Health Educ Res. 2005;20:334-44.

37. Hibbard J, Peters E. Supporting informed consumer health care decisions: data presentation approaches that facilitate the use of information in choice. Annu Rev Public Health. 2003:24:413-33.

38. Doak C, Doak L, Root J. Teaching patients with low literacy skills. 2nd ed. Philadelphia: JB Lippincott Company; 1995.

39. Isaac S, Michael W. Handbook in research and evaluation: a collection of principles, methods, and strategies useful in the planning, design, and evaluation of studies in education and the behavioral sciences. 3rd ed. San Diego: Educational and Industrial Testing Services; 1995.

40. Hill R. What sample size is "enough" in Internet survey research. Interpersonal Computing and Technology: An Electronic Journal for the 21st Century. 1998:6:3-4

41. Julious SA. Sample size of 12 per group rule of thumb for a pilot study. Pharmaceutical Stat. 2005:4:287-91.

42. Van Belle G. Statistical rules of thumb. 2nd ed. New York: John Wiley; 2008.

43. Connelly L. Pilot studies. Medsurg Nurs. 2008;17:411-2.

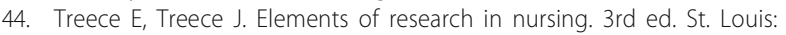
Mosby; 1982.

45. Rowlands G, Khazaezadeh N, Oteng-Ntim E, Seed P, Barr S, Weiss BD. Development and validation of a measure of health literacy in the UK: the newest vital sign. BMC Public Health. 2013;13:116.

\section{Submit your next manuscript to BioMed Central and we will help you at every step:}

- We accept pre-submission inquiries

- Our selector tool helps you to find the most relevant journal

- We provide round the clock customer support

- Convenient online submission

- Thorough peer review

- Inclusion in PubMed and all major indexing services

- Maximum visibility for your research

Submit your manuscript at www.biomedcentral.com/submit 